\title{
Percepciones y habilidades financieras en estudiantes universitarios
}

\author{
William R. Avendaño', Gerson Rueda ${ }^{2}$ y Blanca M. Velasco ${ }^{3}$ \\ (1) Facultad de Ciencias Empresariales, Universidad Francisco de Paula Santander, Cúcuta, Colombia. \\ (correo-e: williamavendano@ufps.edu.co) \\ (2) Facultad de Ciencias Empresariales, Universidad Francisco de Paula Santander, Cúcuta, Colombia. \\ (correo-e: gersonruedavera@ufps.edu.co) \\ (3) Facultad de Ciencias Administrativas y Económicas, Universidad Francisco de Paula Santander, Ocaña, Colombia. \\ (correo-e: bmvelasco@ufpso.edu.co)
}

Recibido Nov. 20, 2020; Aceptado Ene. 18, 2021; Versión final Mar. 13, 2021, Publicado Jun. 2021

\begin{abstract}
Resumen
En el presente estudio se analizan las percepciones y habilidades financieras en un grupo de 307 estudiantes de pregrado de una institución educativa superior pública de la ciudad de Ocaña (Colombia). La investigación tiene un enfoque cuantitativo, no experimental transversal de nivel descriptivo, y se enmarca en el paradigma empírico analítico. Se empleó como instrumento un cuestionario tipo Likert de 33 preguntas divididas en tres dimensiones: 1 ) datos sociodemográficos, 2) percepciones y 3) habilidades-usos. Los datos fueron analizados a través de estadística descriptiva. Los resultados muestran que los estudiantes tienen una percepción y actitud favorable respecto de los temas financieros, aunque se evidencian debilidades en las dimensiones relacionadas con las habilidades-usos financieros. Se concluye que existen limitaciones en el saber-hacer relacionado con la alfabetización financiera y la necesidad del desarrollo de programas específicos focalizados en competencias.
\end{abstract}

Palabras clave: alfabetización financiera; educación; habilidades financieras; percepciones financieras

\section{Financial perceptions and skills among university students}

\begin{abstract}
The present research study analyzes financial perceptions and skills in a group of 307 undergraduate students from a public higher education institution located in the city of Ocaña (Colombia). The approach is quantitative, non-experimental, cross-sectional, and descriptive and is framed within an analytical empirical paradigm. A 33 question Likert-type questionnaire is applied and divided in three dimensions: 1) sociodemographic data, 2) perceptions, and 3) skills-usage. Collected data are analyzed by descriptive statistics. The results show that students have favorable perceptions and attitudes towards financial issues, although weaknesses are evident in the dimensions related to financial skills-usage. It is concluded that there are limitations on financial literacy know-how and that there is a need for developing specific programs that focus on competencies.
\end{abstract}

Keywords: financial literacy; education; financial skills; financial perceptions 


\section{INTRODUCCIÓN}

La educación financiera puede ser interpretada como un proceso, una herramienta o una estrategia, dirigida al desarrollo de conocimientos, actitudes, habilidades, destrezas y comportamientos que facilite la toma de decisiones financieras inteligentes y conscientes conforme a la información que se analiza en materia de riesgos y oportunidades. En ese sentido, en la educación financiera es importante el desarrollo de competencias de diferente tipo que permitan un comportamiento financiero adecuado y la toma de decisiones informadas sobre productos y servicios financieros para la mejora de la calidad de vida y el logro del bienestar social (Duque et al., 2016; Ramos et al., 2017). Villada et al. (2017) agrega que el componente ético es fundamental en la educación financiera y Kiyosaki (2015) manifiesta que en la formación financiera subyacen dos componentes: el alfabetismo financiero entendido como el conocimiento o los saberes financieros, y el IQ financiero comprendido como la capacidad para brindar soluciones a problemas financieros.

En la literatura se destaca la importancia, necesidad y utilidad de la educación financiera porque conlleva a la alfabetización financiera de los sujetos, sin importar el nivel académico y la línea profesional o vocacional por la que se incline la persona. Incluso, desde primaria se agregan algunos temas financieros y contables de manera transversal al área de matemáticas y en formaciones posteriores a la secundaria, se observa que es cada vez más urgente el conocimiento y el desarrollo de habilidades en materia financiera (Fernandes et al., 2014; Lusardi, 2019; Frisancho, 2020). Diferentes investigaciones demuestran que el uso de programas de educación financiera trae efectos positivos sobre los sujetos en las dimensiones del conocimiento, las actitudes, las habilidades y el comportamiento (Kaiser y Menkhoff, 2020; Amagir et al., 2020; Batty et al., 2020).

El éxito de este tipo de programas dependerá de distintos factores, entre estos, la percepción que tienen las personas respecto de la educación financiera y los temas financieros. En efecto, las percepciones regulan y determinan las actitudes, posturas, comportamientos y conductas de los individuos. Las percepciones pueden ser entendidas como proceso y resultado. En el primer escenario, la persona recibe información producto de las fuentes de estímulo, lleva a cabo el tratamiento de la información en un nivel cognoscitivo y emocional, y con ello genera ideas, representaciones, conceptos y símbolos. Ahora bien, las percepciones como resultados, hace referencia a estructuras complejas de comprensión e interpretación de los fenómenos, las realidades y el mundo. Así, múltiples ideas, representaciones, conceptos y símbolos constituirán 'la percepción' que tiene un individuo respecto de una realidad u objeto en particular. La percepción es una visión amplia y compleja que construye la persona y que delimita su modo de vida, su cotidianidad y las interacciones con los demás.

La necesidad de la alfabetización financiera se encuentra ampliamente documentada: los problemas económicos, las crisis financieras, la incidencia de estos temas en el bienestar de las personas y las familias, el papel protagónico que ahora tienen los consumidores, el aumento de la esperanza de vida, la relevancia de las pensiones, la complejidad de los actuales productos financieros, entre otros (Lusardi, 2019; Sánchez et al., 2020; Garay, 2016). Sin duda, la educación financiera es un aspecto clave en la formación de las personas, y por ello, se relaciona con el mismo ejercicio de la ciudadanía. En ese sentido, las instituciones educativas deben generar programas y cursos que permitan el fortalecimiento de las capacidades y las habilidades financieras entre los sujetos, pero siempre considerando un modelo pedagógico idóneo para ello. Considerando la naturaleza de los saberes relacionados con este campo y el contexto en el que giran, un enfoque crítico parece el más idóneo para el desarrollo de capacidades críticas en el marco de la alfabetización financiera (Carvajal et al., 2016).

Se debe interpretar la educación financiera como una herramienta que puede apoyar los procesos formativos en diferentes áreas del conocimiento, así como en la inclusión social de los ciudadanos y la estabilidad de los mercados financieros (Grohmann et al., 2018; Connolly y Khaldoun, 2001). Dadas las oportunidades y los beneficios de la alfabetización financiera, es que en muchos contextos se han diseñado políticas públicas educativas o estrategias educativas con el objetivo de regular y organizar la educación financiera en los diferentes niveles de escolaridad. En Colombia, por ejemplo, hay un documento expedido por el Ministerio de Educación Nacional (2014) en el que se orienta la incorporación de la educación económica y financiera de forma transversal al área de ciencias sociales en los niveles formales de primaria, secundaria y media. Pero ello, puede desconocer la importancia que tienen otras áreas en la formación financiera de un joven o minimizar el campo de saber de las finanzas. Frente a esta situación, se promueve en la actualidad una iniciativa legislativa en el Congreso de la República para que se establezca la Cátedra Obligatoria de Educación Económica y Financiera en todas las instituciones educativas de Colombia a través del Proyecto de Ley 222/2019 Senado. 
En el terreno universitario, también se muestra que la alfabetización financiera es un área que requiere un mayor desarrollo y fortalecimiento. La literatura describe que en América Latina, incluso, países de Europa o Estados Unidos, la formación financiera de los universitarios resulta escasa, al igual que sus conocimientos y habilidades en diferentes variables, lo que plantea retos y desafíos tanto institucionales como estatales (Garay, 2016; Moreno et al., 2017). En Colombia, son escasos los estudios relacionados con las percepciones, habilidades y usos financieros de estudiantes universitarios (Silva, et al., 2018; Moreno et al., 2017; Carvajal et al., 2016).

Por otro lado, se deben considerar algunos indicadores que demuestran la necesidad de una alfabetización financiera en el contexto colombiano: 1) uno de cada diez colombianos ahorra; 2) el 9\% de adultos ahorran en instituciones bancarias y el $18 \%$ de manera informal; 3 ) el $22 \%$ de los hogares gasta más del presupuesto disponible; 4) el nivel de morosidad es alto entre los usuarios y clientes; y 5) los jóvenes entre 18 y 25 años son los que menos tienen relaciones con el sector bancario y financiero (Superintendencia Financiera de Colombia, 2019; Federación Latinoamericana de Bancos, 2019).

El objetivo de esta investigación es analizar las percepciones y habilidades-usos financieras en un grupo de estudiantes de pregrado en una institución educativa superior de la ciudad de Ocaña en Colombia. Los resultados de esta investigación se pueden sumar a los escasos estudios realizados en el país sobre este tema y evidenciar puntos clave que deberían tener en cuenta en el diseño de una política o estrategia bien macro o de tipo institucional. En efecto, el punto de partida en la formulación de acciones estratégicas en cualquier nivel es la información objetiva que se cuenta sobre el problema. En este estudio, se muestra la realidad de 307 participantes de pregrado.

\section{METODOLOGÍA}

La investigación tiene un enfoque cuantitativo y se enmarca en el paradigma empírico-analítico. De este modo, el método empleado es deductivo y el tipo de estudio es no experimental-transversal de nivel descriptivo. La población estuvo integrada por 1.514 estudiantes de los programas académicos de la Facultad de Ciencias Administrativas y Económicas de la Universidad Francisco de Paula Santander Sede Ocaña en Colombia: 523 estudiantes del programa de Administración de Empresas, 725 del programa de Contaduría Pública y 266 del programa de Tecnología en Gestión Comercial y Financiera. Se aplicó la siguiente fórmula estadística para determinar la muestra a través del método aleatorio simple:

$$
\mathrm{n}=\frac{Z c^{2} \times P \times Q \times}{E^{2} \times(N-1)+Z c^{2} \times P \times Q}
$$

Donde: $Z c=95 \%$ o 1.96 es el nivel de certeza, bajo la curva normal; $P=0,5$ es la probabilidad de éxito; $Q=$ 0,5 es la probabilidad de fracaso; $E=5 \%-0,05$ es el nivel de error; y $N=1.514$ es la población. Aplicada la fórmula estadística, se calcula la muestra en 307 estudiantes de pregrado de la Facultad ya mencionada. La muestra estuvo dividida de la siguiente manera: 183 estudiantes del programa Administración de Empresas (59,61\%), 78 estudiantes del programa Contaduría Pública (25,41\%) y 46 estudiantes del programa Tecnología en Gestión Comercial y Financiera (14,98\%). Se empleó como instrumento un cuestionario tipo Likert con un total de 33 preguntas para una única variable nominal (percepciones y habilidades financieras en estudiantes universitarios) con las dimensiones e indicadores que se describen en la Tabla 1.

En general, se revisó una sola variable como ya se indicó con tres dimensiones: datos sociodemográficos, percepción sobre temas financieros y habilidad financiera - uso financiero. En cada una de las dimensiones, excepto la de datos sociodemográficos, se plantearon preguntas con afirmaciones y opciones de respuesta de la siguiente manera: muy de acuerdo, algo de acuerdo, ni de acuerdo ni en desacuerdo, algo en desacuerdo y muy en desacuerdo.

La validación y confiabilidad del instrumento se determinó a través de juicio de expertos y el Alfa de Cronbach. Para la primera técnica, participaron en este proceso cuatro expertos en el campo de la educación y un experto en temas financieros, todos con doctorado, publicaciones científicas y experiencia superior a los 15 años. Por otro lado, el Alfa de Cronbach para el instrumento obtuvo un coeficiente de confiabilidad de 0,89. A raíz de la situación generada por la COVID-19 y las recomendaciones de distanciamiento social, el cuestionario fue aplicado a los estudiantes a través de la plataforma Formularios de Google. A los estudiantes se les informó sobre el alcance y objetivos de la investigación y el instrumento, y que este solo tenía fines académicos. Estas explicaciones también se dejaron claras dentro de Google Forms, y por tanto, los participantes mostraban consentimiento libre e informado sobre la encuesta a la que darían respuesta. Los datos recolectados fueron organizados, tabulados y analizados de manera individual, y luego, mediante un análisis estadístico a través del Baremo descrito en la Tabla 2. 
Tabla 1: Estructura del cuestionario aplicado a jóvenes universitarios de la Facultad de Ciencias Administrativas y Económicas de la UFPS (Cúcuta, Colombia)

\begin{tabular}{|c|c|c|}
\hline Dimensiones & Indicadores & Preguntas \\
\hline \multirow{5}{*}{ Sociodemográfico } & Género & \multirow{5}{*}{$1-7$} \\
\hline & Edad & \\
\hline & Estrato socioeconómico & \\
\hline & Programa & \\
\hline & Residencia - Sector & \\
\hline \multirow{15}{*}{ Percepción sobre temas financieros } & Importancia & \multirow{5}{*}{ 8-12 } \\
\hline & Utilidad & \\
\hline & Actitud & \\
\hline & Importancia transmitida en la institución educativa & \\
\hline & Importancia transmitida en casa & \\
\hline & Ahorro & \multirow{10}{*}{$13-22$} \\
\hline & Inversión & \\
\hline & Tasa de retorno & \\
\hline & Tarjetas de crédito & \\
\hline & Intereses & \\
\hline & Inflación & \\
\hline & Créditos & \\
\hline & Seguros & \\
\hline & Riesgo financiero & \\
\hline & Planeación financiera & \\
\hline \multirow{3}{*}{ Habilidad financiera y uso financiero } & Acciones de planificación & \multirow{3}{*}{$23-33$} \\
\hline & Acciones de análisis & \\
\hline & Comportamiento financiero & \\
\hline
\end{tabular}

Tabla 2: Resultados de la dimensión percepciones sobre los temas financieros

\begin{tabular}{|c|c|c|}
\hline Rango de actuación & Categorías & Criterios \\
\hline $1-1,99$ & Muy en desacuerdo & Muy baja \\
\hline $2-2,99$ & Algo en desacuerdo & Baja \\
\hline $3-3,99$ & Ni de acuerdo ni en desacuerdo & Moderada \\
\hline $4-4,99$ & Algo de acuerdo & Alta \\
\hline 5 & Muy de acuerdo & Muy alta \\
\hline
\end{tabular}

\section{RESULTADOS}

Las características sociodemográficas de la muestra se exponen en la Tabla 2 . El $67.4 \%$ de los participantes correspondieron a mujeres y el mayor número de encuestados se encuentran según edad, entre los 15 y 25 años de edad (89.2\%). Igualmente, el estrato socioeconómico que predomina entre la muestra es 1 y 2 (94.3\%) y no se evidencia una predominancia significativa según semestre. El $51.7 \%$ se encuentran entre los semestres 3 y 6 . El $85.9 \%$ tiene su residencia en zona urbana. En la Tabla 3 se exponen los resultados de la dimensión percepciones sobre los temas financieros, la cual explora aspectos como la importancia, actitud e interés que otorgan los participantes a los tópicos financieros, además, si en 
entornos como el hogar o la universidad reciben información sobre este tipo de temas. Se logra identificar que, en su mayoría, los estudiantes, le dan una importancia significativa (95.8\%) a temas como dinero, ahorro, inversión, tasa de retorno y otros similares (68.8\% muy de acuerdo y $27.0 \%$ algo de acuerdo). El $83.71 \%$ considera que es útil tener conocimientos sobre temas financieros y alrededor del $96.4 \%$ señala informarse y aprender sobre este tipo de asuntos (68.4\% muy de acuerdo y $28.0 \%$ algo de acuerdo).

Tabla 3: Resultados de la dimensión percepciones sobre los temas financieros

\begin{tabular}{|c|c|c|c|}
\hline Pregunta & Opciones & No & $\%$ \\
\hline \multirow{5}{*}{$\begin{array}{l}\text { Le doy bastante importancia a los temas financieros como como } \\
\text { dinero, ahorro, imversión, tasa de retorno, tarjetas de crédito, } \\
\text { intereses, inflación, créditos, seguros, riesgo y otros similares }\end{array}$} & Muy de acuerdo & 209 & 68.0 \\
\hline & Algo de acuerdo & 83 & 27.0 \\
\hline & Ni de acuerdo ni en desacuerdo & 11 & 3.5 \\
\hline & Algo en desacuerdo & 3 & 0.9 \\
\hline & Muy en desacuerdo & 1 & 0.3 \\
\hline \multirow{5}{*}{$\begin{array}{l}\text { Considero que conocer sobre temas financieros [...]; resulta bastante } \\
\text { útil para mi vida. }\end{array}$} & Muy de acuerdo & 257 & 83.7 \\
\hline & Algo de acuerdo & 42 & 13.6 \\
\hline & Ni de acuerdo ni en desacuerdo & 4 & 1.3 \\
\hline & Algo en desacuerdo & 4 & 1.3 \\
\hline & Muy en desacuerdo & 0 & 0 \\
\hline \multirow{5}{*}{$\begin{array}{l}\text { Cuando se está informando sobre temas financieros [...]; procuro } \\
\text { prestar atención y aprender sobre los mismos. }\end{array}$} & Muy de acuerdo & 210 & 68.4 \\
\hline & Algo de acuerdo & 86 & 28.0 \\
\hline & Ni de acuerdo ni en desacuerdo & 8 & 2.6 \\
\hline & Algo en desacuerdo & 1 & 0.3 \\
\hline & Muy en desacuerdo & 2 & 0.6 \\
\hline \multirow{5}{*}{$\begin{array}{l}\text { En clases, los profesores procuran explicar la importancia de temas } \\
\text { financieros }[\ldots] .\end{array}$} & Muy de acuerdo & 140 & 45.6 \\
\hline & Algo de acuerdo & 124 & 40.3 \\
\hline & Ni de acuerdo ni en desacuerdo & 24 & 7.8 \\
\hline & Algo en desacuerdo & 16 & 5.2 \\
\hline & Muy en desacuerdo & 3 & 0.9 \\
\hline \multirow{5}{*}{$\begin{array}{l}\text { En casa, mis padres o familiares procuran explicar la importancia de } \\
\text { temas financieros }[\ldots] \text {. }\end{array}$} & Muy de acuerdo & 121 & 39.4 \\
\hline & Algo de acuerdo & 106 & 34.5 \\
\hline & Ni de acuerdo ni en desacuerdo & 45 & 14.6 \\
\hline & Algo en desacuerdo & 28 & 9.1 \\
\hline & Muy en desacuerdo & 7 & 2.2 \\
\hline \multirow{5}{*}{$\begin{array}{l}\text { Conozco de manera clara que es el ahorro, sus características y la } \\
\text { forma en que opera en la práctica. }\end{array}$} & Muy de acuerdo & 140 & 45.6 \\
\hline & Algo de acuerdo & 134 & 43.6 \\
\hline & Ni de acuerdo ni en desacuerdo & 19 & 6.1 \\
\hline & Algo en desacuerdo & 13 & 4.2 \\
\hline & Muy en desacuerdo & 1 & 0.3 \\
\hline \multirow{5}{*}{$\begin{array}{l}\text { Conozco de manera clara que es la inversión, sus características y la } \\
\text { forma en que opera en la práctica. }\end{array}$} & Muy de acuerdo & 128 & 41.6 \\
\hline & Algo de acuerdo & 136 & 44.3 \\
\hline & Ni de acuerdo ni en desacuerdo & 22 & 7.1 \\
\hline & Algo en desacuerdo & 18 & 5.8 \\
\hline & Muy en desacuerdo & 3 & 0.9 \\
\hline \multirow{5}{*}{$\begin{array}{l}\text { Conozco de manera clara que es la tasa de retorno, sus } \\
\text { características y la forma en que opera en la práctica. }\end{array}$} & Muy de acuerdo & 56 & 18.2 \\
\hline & Algo de acuerdo & 146 & 47.5 \\
\hline & Ni de acuerdo ni en desacuerdo & 55 & 17.9 \\
\hline & Algo en desacuerdo & 35 & 11.4 \\
\hline & Muy en desacuerdo & 15 & 4.8 \\
\hline \multirow{5}{*}{$\begin{array}{l}\text { Conozco de manera clara que son las tarjetas de crédito, sus } \\
\text { características y la forma en que opera en la práctica. }\end{array}$} & Muy de acuerdo & 109 & 35.5 \\
\hline & Algo de acuerdo & 139 & 45.2 \\
\hline & Ni de acuerdo ni en desacuerdo & 29 & 9.4 \\
\hline & Algo en desacuerdo & 21 & 6.8 \\
\hline & Muy en desacuerdo & 9 & 2.9 \\
\hline \multirow{5}{*}{$\begin{array}{l}\text { Conozco de manera clara que son los intereses, sus características } \\
\text { y la forma en que opera en la práctica. }\end{array}$} & Muy de acuerdo & 120 & 39.0 \\
\hline & Algo de acuerdo & 145 & 47.2 \\
\hline & Ni de acuerdo ni en desacuerdo & 27 & 8.8 \\
\hline & Algo en desacuerdo & 13 & 4.2 \\
\hline & Muy en desacuerdo & 2 & 0.6 \\
\hline \multirow{5}{*}{$\begin{array}{l}\text { Conozco de manera clara que es la inflación, sus características y la } \\
\text { forma en que opera en la práctica. }\end{array}$} & Muy de acuerdo & 114 & 37.1 \\
\hline & Algo de acuerdo & 146 & 47.5 \\
\hline & Ni de acuerdo ni en desacuerdo & 29 & 9.4 \\
\hline & Algo en desacuerdo & 14 & 4.5 \\
\hline & Muy en desacuerdo & 4 & 1.3 \\
\hline
\end{tabular}


Tabla 3: continuación.

\begin{tabular}{|c|c|c|c|}
\hline Pregunta & Opciones & No & $\%$ \\
\hline \multirow{5}{*}{$\begin{array}{l}\text { Conozco de manera clara que son los créditos, sus características y } \\
\text { la forma en que opera en la práctica. }\end{array}$} & Muy de acuerdo & 125 & 40.7 \\
\hline & Algo de acuerdo & 148 & 48.2 \\
\hline & Ni de acuerdo ni en desacuerdo & 26 & 8.4 \\
\hline & Algo en desacuerdo & 7 & 2.2 \\
\hline & Muy en desacuerdo & 1 & 0.3 \\
\hline \multirow{5}{*}{$\begin{array}{l}\text { Conozco de manera clara que son los seguros, sus características y } \\
\text { la forma en que opera en la práctica. }\end{array}$} & Muy de acuerdo & 91 & 29.6 \\
\hline & Algo de acuerdo & 156 & 50.8 \\
\hline & Ni de acuerdo ni en desacuerdo & 42 & 13.6 \\
\hline & Algo en desacuerdo & 15 & 4.8 \\
\hline & Muy en desacuerdo & 3 & 0.9 \\
\hline \multirow{5}{*}{$\begin{array}{l}\text { Conozco de manera clara que es el riesgo financiero, sus } \\
\text { características y la forma en que opera en la práctica. }\end{array}$} & Muy de acuerdo & 80 & 26.0 \\
\hline & Algo de acuerdo & 142 & 46.2 \\
\hline & $\mathrm{Ni}$ de acuerdo ni en desacuerdo & 55 & 17.9 \\
\hline & Algo en desacuerdo & 23 & 7.4 \\
\hline & Muy en desacuerdo & 7 & 2.2 \\
\hline \multirow{5}{*}{$\begin{array}{l}\text { Conozco de manera clara que es la planeación financiera, sus } \\
\text { características y la forma en que opera en la práctica. }\end{array}$} & Muy de acuerdo & 71 & 23.1 \\
\hline & Algo de acuerdo & 166 & 54.0 \\
\hline & Ni de acuerdo ni en desacuerdo & 44 & 14.3 \\
\hline & Algo en desacuerdo & 19 & 6.1 \\
\hline & Muy en desacuerdo & 7 & 2.2 \\
\hline
\end{tabular}

Respecto a sus entornos hogar o universidad, las respuestas permiten evidenciar que en ambos contextos existe un interés significativo por los temas financieros y buscan promocionarlo: $85.9 \%$ de los participantes están muy de acuerdo o algo de acuerdo en el caso de la promoción de los temas financieros en la universidad y el $73.9 \%$ de los estudiantes están muy de acuerdo o algo de acuerdo en el caso de la promoción de la promoción de los temas financieros en el hogar.

Los resultados también permiten evidenciar que los estudiantes, a partir de su auto-percepción sobre la apropiación o manejo de saberes financieros, poco manejan temas como la planeación financiera, el riesgo financiero, los seguros, la inflación, los intereses, las tarjetas de crédito y la tasa de retorno. Al indagar por la percepción que tenían sobre dichos temas financieros, menos del $45.0 \%$ para todos los ítems admitió saber con claridad sus características y la forma en que opera en la práctica (para todas las afirmaciones los porcentajes de las respuestas variaban entre $45.6 \%$ y $18.2 \%$ para la opción "muy de acuerdo"). En temas como ahorro, inversión y créditos mostraron una auto-percepción más aceptables, sin embargo, ninguno de los ítems supero el $50 \%$ de respuestas para "muy acuerdo", lo que indica que los estudiantes perciben que no manejan a profundidad y de manera sólida este tipo de temas y la forma en que operan dentro de la realidad.

En la Tabla 4 se muestran los hallazgos para la dimensión habilidad financiera y uso financiero. Respecto de habilidades de planificación financiera se evidencia que menos del $60 \%$ planea el pago de sus obligaciones y alrededor del $42 \%$ organiza un presupuesto. Por tanto, un porcentaje similar $(44.9 \%)$ son los que afirman establecer metas financieras a diferentes plazos de tiempo. Aunque al parecer cerca del $87 \%$ de los encuestados posee productos crediticios, existe una débil organización entre ingresos de la persona y uso de dichos productos, y pese a que se identificó que el $50.4 \%$ proyecta adquisición de grandes bienes como vivienda u automóvil, la falta de organización y planeación podrían dificultar la consecución del objetivo.

Tabla 4: Resultados de la dimensión habilidad financiera y uso financiero

\begin{tabular}{|c|c|c|c|}
\hline Pregunta & Opciones & No & $\%$ \\
\hline \multirow{5}{*}{$\begin{array}{l}\text { Planifico el pago de mis deudas, } \\
\text { facturas y/o créditos }\end{array}$} & Muy de acuerdo & 182 & 59.2 \\
\hline & Algo de acuerdo & 96 & 31.2 \\
\hline & Ni de acuerdo ni en desacuerdo & 20 & 6.5 \\
\hline & Algo en desacuerdo & 5 & 1.6 \\
\hline & Muy en desacuerdo & 4 & 1.3 \\
\hline \multirow{5}{*}{$\begin{array}{l}\text { Establezco metas financieras a corto } \\
\text { plazo (semanas), mediano plazo } \\
\text { (meses) y largo plazo (+ de un año) }\end{array}$} & Muy de acuerdo & 138 & 44.9 \\
\hline & Algo de acuerdo & 120 & 39.0 \\
\hline & Ni de acuerdo ni en desacuerdo & 32 & 10.4 \\
\hline & Algo en desacuerdo & 12 & 3.9 \\
\hline & Muy en desacuerdo & 5 & 1.6 \\
\hline
\end{tabular}


Tabla 4: continuación.

\begin{tabular}{|c|c|c|c|}
\hline Pregunta & Opciones & No & $\%$ \\
\hline \multirow{5}{*}{$\begin{array}{l}\text { Elaboro presupuestos (ingresos y } \\
\text { egresos) para llevar a cabo un } \\
\text { comportamiento organizado y serio } \\
\text { sobre mis finanzas. }\end{array}$} & Muy de acuerdo & 130 & 42.3 \\
\hline & Algo de acuerdo & 117 & 38.1 \\
\hline & $\mathrm{Ni}$ de acuerdo ni en desacuerdo & 41 & 13.3 \\
\hline & Algo en desacuerdo & 14 & 4.5 \\
\hline & Muy en desacuerdo & 5 & 1.6 \\
\hline \multirow{6}{*}{$\begin{array}{l}\text { En caso de tener productos financieros } \\
\text { como tarjetas de crédito o créditos, } \\
\text { planificó su uso conforme a mis } \\
\text { ingresos. }\end{array}$} & Muy de acuerdo & 132 & 43.0 \\
\hline & Algo de acuerdo & 83 & 27.0 \\
\hline & Ni de acuerdo ni en desacuerdo & 40 & 13.0 \\
\hline & Algo en desacuerdo & 8 & 2.6 \\
\hline & Muy en desacuerdo & 7 & 2.2 \\
\hline & No tengo ni manejo tarjetas de crédito o débito & 37 & 12.0 \\
\hline \multirow{5}{*}{$\begin{array}{l}\text { Desde ahora, ya he pensado la forma } \\
\text { de organizar mis finanzas para compras } \\
\text { significativas como vivienda, automóvil u } \\
\text { otro similar. }\end{array}$} & Muy de acuerdo & 155 & 50.4 \\
\hline & Algo de acuerdo & 101 & 32.9 \\
\hline & $\mathrm{Ni}$ de acuerdo ni en desacuerdo & 28 & 9.1 \\
\hline & Algo en desacuerdo & 13 & 4.2 \\
\hline & Muy en desacuerdo & 10 & 3.2 \\
\hline \multirow{5}{*}{$\begin{array}{l}\text { Analizó todas las compras que realizo } \\
\text { examinando las implicaciones que ello } \\
\text { trae a mis finanzas personales. }\end{array}$} & Muy de acuerdo & 143 & 46.5 \\
\hline & Algo de acuerdo & 117 & 38.1 \\
\hline & Ni de acuerdo ni en desacuerdo & 34 & 11.0 \\
\hline & Algo en desacuerdo & 12 & 3.9 \\
\hline & Muy en desacuerdo & 1 & 0.3 \\
\hline \multirow{6}{*}{$\begin{array}{l}\text { En caso de tener productos financieros } \\
\text { como tarjetas de crédito o créditos, } \\
\text { analizo comprensivamente los intereses, } \\
\text { tiempos y otras variables. }\end{array}$} & Muy de acuerdo & 107 & 34.8 \\
\hline & Algo de acuerdo & 110 & 35.8 \\
\hline & Ni de acuerdo ni en desacuerdo & 34 & 11.0 \\
\hline & Algo en desacuerdo & 8 & 2.6 \\
\hline & Muy en desacuerdo & 8 & 2.6 \\
\hline & No tengo ni manejo tarjetas de crédito o créditos & 40 & 13.0 \\
\hline \multirow{5}{*}{$\begin{array}{l}\text { Vigilo el tema de mis finanzas } \\
\text { personales y tomo decisiones a partir de } \\
\text { la información que tengo. }\end{array}$} & Muy de acuerdo & 149 & 48.5 \\
\hline & Algo de acuerdo & 121 & 39.4 \\
\hline & $\mathrm{Ni}$ de acuerdo ni en desacuerdo & 26 & 8.4 \\
\hline & Algo en desacuerdo & 9 & 2.9 \\
\hline & Muy en desacuerdo & 2 & 0.6 \\
\hline \multirow{5}{*}{$\begin{array}{l}\text { Ahorro de manera permanente conforme } \\
\text { a metas que me he planteado. }\end{array}$} & Muy de acuerdo & 152 & 49.5 \\
\hline & Algo de acuerdo & 111 & 36.1 \\
\hline & $\mathrm{Ni}$ de acuerdo ni en desacuerdo & 25 & 8.1 \\
\hline & Algo en desacuerdo & 13 & 4.2 \\
\hline & Muy en desacuerdo & 6 & 1.9 \\
\hline \multirow{5}{*}{$\begin{array}{l}\text { Pago mis deudas, facturas y/o créditos } \\
\text { siempre a tiempo. }\end{array}$} & Muy de acuerdo & 203 & 66.1 \\
\hline & Algo de acuerdo & 79 & 25.7 \\
\hline & Ni de acuerdo ni en desacuerdo & 17 & 5.5 \\
\hline & Algo en desacuerdo & 7 & 2.2 \\
\hline & Muy en desacuerdo & 1 & 0.3 \\
\hline \multirow{5}{*}{$\begin{array}{l}\text { Conozco mi nivel de endeudamiento, y a } \\
\text { partir de ello, tomo decisiones } \\
\text { financieras. }\end{array}$} & Muy de acuerdo & 197 & 64.1 \\
\hline & Algo de acuerdo & 84 & 27.3 \\
\hline & $\mathrm{Ni}$ de acuerdo ni en desacuerdo & 15 & 4.8 \\
\hline & Algo en desacuerdo & 7 & 2.2 \\
\hline & Muy en desacuerdo & 4 & 1.3 \\
\hline
\end{tabular}

En lo referente a acciones de análisis para organizar y llevar una vida financiera saludable, se logra identificar que no es muy común analizar las implicaciones financieras de realizar determinada compra en efectivo, o los intereses y demás cobros adicionales que significa realizar una adquisición de un bien o servicio mediante un crédito. La vigilancia sobre el tema de las finanzas personales es algo con lo que menos de la mitad de los encuestados están muy de acuerdo (48.5\%). Respecto a temas como ahorro, responsabilidad en el pago de las obligaciones y conocimiento del nivel de endeudamiento, los encuestados se mostraron muy de acuerdo en $49.5 \%, 66.1 \%$ y $64.1 \%$, respectivamente. Esto indicaría un comportamiento adecuado más no relevante paras el grupo perteneciente a la muestra.

Obtenidos los resultados anteriores, se realizó un tratamiento estadístico a partir de las medias obtenidas y conforme al baremo sugerido. Los hallazgos del análisis estadístico se muestran en la Tabla 5 y sugieren una media alta en cada una de las dimensiones con debilidades en indicadores específicos pero que no influyen en la media obtenida en general. 
Tabla 5: Resultados de la dimensión habilidad financiera y uso financiero

\begin{tabular}{|c|c|c|c|c|c|c|c|}
\hline Ítem & Media & Indicador & Indicador & Criterio & Dimensión & Media & Criterio \\
\hline 6 & 4,61 & Importancia personal & 4,61 & Alta & \multirow{5}{*}{$\begin{array}{l}\text { Percepción sobre temas } \\
\text { financieros }\end{array}$} & \multirow{5}{*}{4,46} & \multirow{5}{*}{ Alta } \\
\hline 7 & 4,80 & Utilidad & 4,80 & Alta & & & \\
\hline 8 & 4,64 & Actitud & 4,64 & Alta & & & \\
\hline 9 & 4,26 & Importancia desde IE & 4,26 & Alta & & & \\
\hline 10 & 4,02 & Importancia desde casa & 4,02 & Alta & & & \\
\hline 11 & 4,33 & Ahorro & 4,33 & Alta & \multirow{10}{*}{$\begin{array}{l}\text { Percepción sobre temas } \\
\text { financieros específicos }\end{array}$} & \multirow{10}{*}{4,11} & \multirow{10}{*}{ Alta } \\
\hline 12 & 4,23 & Inversión & 4,23 & Alta & & & \\
\hline 13 & 3,67 & Tasa de retorno & 3,67 & Moderada & & & \\
\hline 14 & 4,08 & Tarjetas de crédito & 4,08 & Alta & & & \\
\hline 15 & 4,25 & Intereses & 4,25 & Alta & & & \\
\hline 16 & 4,21 & Inflación & 4,21 & Alta & & & \\
\hline 17 & 4,33 & Créditos & 4,33 & Alta & & & \\
\hline 18 & 4,11 & Seguros & 4,11 & Alta & & & \\
\hline 19 & 3,94 & Riesgo financiero & 3,94 & Moderada & & & \\
\hline 20 & 3,98 & Planeación financiera & 3,98 & Moderada & & & \\
\hline 21 & 4,55 & \multirow{5}{*}{ Acciones de planificación } & \multirow{5}{*}{4,33} & \multirow{5}{*}{ Alta } & \multirow{11}{*}{$\begin{array}{l}\text { Habilidad financiera y } \\
\text { uso financiero }\end{array}$} & \multirow{11}{*}{4,38} & \multirow{11}{*}{ Alta } \\
\hline 22 & 4,32 & & & & & & \\
\hline 23 & 4,26 & & & & & & \\
\hline 24 & 4,17 & & & & & & \\
\hline 25 & 4,35 & & & & & & \\
\hline 26 & 4,39 & \multirow{3}{*}{ Acciones de análisis } & \multirow{3}{*}{4,39} & \multirow{3}{*}{ Alta } & & & \\
\hline 27 & 4,11 & & & & & & \\
\hline 28 & 4,46 & & & & & & \\
\hline 29 & 4,41 & \multirow{3}{*}{ Comportamiento } & \multirow{3}{*}{4,41} & \multirow{3}{*}{ Alta } & & & \\
\hline 30 & 4,70 & & & & & & \\
\hline 31 & 4,71 & & & & & & \\
\hline
\end{tabular}

\section{DISCUSIÓN}

Alrededor del $83.7 \%$ de los participantes en el estudio consideran que conocer sobre temas financieros resulta bastante útil para la vida. Estos hallazgos son contrarios a los obtenidos en otras investigaciones como la de Duque et al. (2016). El estudio en mención, caracteriza las capacidades financieras de 550 jóvenes universitarios en Medellín, pertenecientes a programas de mercadeo, finanzas y negocios internacionales, y allí se describe que estos otorgan escasa importancia a los temas financieros. Sin embargo, es coherente con los hallazgos obtenidos en esta investigación, al evidenciar que las capacidades financieras son bajas entre la población objeto de análisis.

En efecto, este estudio muestra desde las percepciones de los participantes, que hay manejo sobre determinados temas financieros básicos y una actitud positiva en general, pero del mismo modo, debilidades y limitaciones en habilidades y usos financieros más específicos, lo que concuerda con la investigación de Lusardi (2019) para quien existen enormes vulnerabilidades en algunos subgrupos y menor conocimiento en tópicos más puntuales. Lo mismo se demuestra en el estudio de Amagir et al. (2020) donde los estudiantes tienen niveles bajos de educación financiera, especialmente, en grupos de inmigrantes, estatus socioeconómico bajo y con padres sin título universitario.

El saber financiero es esencial para el desarrollo de capacidades financieras, y aplicados, deberían ayudar a mitigar los riesgos respecto de la economía personal o familiar, impulsar una mejor administración de los recursos que se tienen a disposición (Saldarriaga, 2018), e incluso, favorecer los emprendimientos creativos de las personas (Abad y González, 2019). Según las respuestas a la encuesta aplicada en esta investigación, $96.4 \%$ procuran informarse sobre temas financieros para aprender sobre los mismos, y alrededor del $68 \%$ les da una alta importancia, lo que podría interpretarse como positivo en cuanto a lo referente a las actitudes frente a la alfabetización financiera.

La evidencia que dan las respuestas indica que solo el $45.6 \%$ conoce de manera clara que es el ahorro, sus características y la forma en que opera en la práctica, lo cual es un porcentaje bajo. Roa et al., (2018) señalan que tener un nivel más alto de alfabetización financiera disminuye la probabilidad de que un individuo fracase financieramente, además, este tipo de formación es positivamente significativa para 
mantener productos financieros formales como el ahorro. Los autores señalan que las personas que no tienen capacidad para el ahorro poseen una dificultad intrínseca para desarrollar otras capacidades financieras.

Saldarriaga (2018) indica que el instrumento clave para apoyarse y empezar con una cultura de ahorro es el presupuesto, ya que permite a las personas conocer los ingresos y egresos de dinero y llevar a cabo un comportamiento organizado y serio sobre las finanzas. Lo evidenciado en las respuestas respecto de la planeación financiera indica que $42,34 \%$ elaboran un presupuesto para la organización y control de sus finanzas, y $44,95 \%$ establece metas financieras a corto mediano y largo plazo. La falta de control y programación mediante un presupuesto puede dificultar la consecución de ciertos objetivos financieros. Harrington et al., (2014) señala que mediante un presupuesto bien organizado es posible gastar, ahorrar y proyectarse de manera exitosa.

Según las respuestas de las encuestados, solo el $12.0 \%$ no tiene tarjeta de crédito lo que permite estimar que más del $85.0 \%$ de la muestra cuenta con créditos bancarios o con alguna entidad. Cabe tener en cuenta que las tarjetas de crédito es que uno de los productos financieros que más reportes negativos genera en las centrales de riesgo en Colombia, junto con créditos con entidades no financieras y empresas de telecomunicaciones (Arango y Cardona, 2019). Las respuestas entregadas por las personas participantes en el estudio podrían brindar una explicación a lo descrito anteriormente, ya que menos del $50 \%$ planifica el uso de créditos conforme a sus ingresos. Solo el $34.8 \%$ analiza comprensivamente los intereses, tiempos y otras variables referentes al uso de créditos para la adquisición de productos o servicios, y el $46.5 \%$ analiza las implicaciones que trae a las finanzas personales las compras realizadas.

En algunos estudios se muestra que determinados factores llevan a una mejora del comportamiento y la actitud financiera, por ejemplo, la satisfacción en general sobre la vida (Shim et al., 2009). Es sustancial que la información respecto a temas financieros sea analizada, razonada e interpretada según sus individualidades para que, al hacer una elección con respecto a las finanzas, el impacto sea positivo y encaminado hacia el éxito (Xiao y O'Neill, 2016; Fan y Chatterjee, 2019), y frente a ello, programas específicos para el desarrollo de competencias y habilidades financiera puede ser una opción potencial (Iterbeke, et al., 2020; Kaiser y Menkhoff, 2017). Sin embargo, es importante que estos programas sean adelantados por docentes expertos y preparados en el campo para que los resultados sean positivos (Urban, et al., 2020).

\section{CONCLUSIONES}

Los estudiantes participantes del estudio, aunque señalan un interés por conocer, aprender y explorar temas financieros, además de obtener en cada una de las dimensiones un nivel alto en promedio, muestran limitaciones en las dimensiones de percepciones para algunos tópicos específicos y en habilidades-usos financieros. En efecto, se evidencian debilidades en temas como los intereses de mora, los intereses corrientes, las cuotas de manejo, créditos rotativos, entre otros. De esta manera, se exige una educación financiera desde la formación de profesionales que se centre en competencias, esto es, el saber hacer con una amplia socialización de saberes y herramientas aplicables en contexto.

La educación financiera es un proceso e instrumento clave para el desarrollo de competencias financieras, Un individuo que no esté interesado en educarse financieramente está más propenso a vivir de forma precaria en cuanto a su economía. Existen competencias financieras básicas que apoyan el desarrollo de otras, por ejemplo, el hábito de ahorro o el manejo de herramientas como el presupuesto. Conocer, manejar y aplicar metodologías de ahorro o por su parte, planear, gastar, pagar, entre otras posibilidades, mediante un presupuesto, son competencias básicas respecto de una vida financiera saludable.

\section{REFERENCIAS}

Abad, E., y González, M.D., Effects of financial education and financial literacy on creative entrepreneurship: a worldwide research, https://doi.org/10.3390/educsci9030238, Educ. Sci., 9(3), 238-254 (2019)

Amagir, A., Groot, W., Maassen, H., y Wilshut, A., Financial literacy of high school students in the Netherlands: knowledge, attitudes, self-efficacy, and behavior, https://doi.org/10.1016/j.iree.2020.100185, International Review of Economics Education, 34, 1-15 (2020)

Arango L.E., y Cardona, L., Tarjetas de crédito en personas de ingresos medios y bajos en Colombia: ¿qué determina su uso?, https://doi.org/10.32468/be.1089, Borradores de Economía, (1089), 1-46 (2019)

Batty, M., Collins, M., O’Rourke, C., y Odders, E., Experiential financial education: a field study of my classroom economy in elementary schools, https://doi.org/10.1016/j.econedurev.2020.102014, Economics of Education Review, 78, $1-11(2020)$ 
Connolly, C., y Khaldoun, H., Financial services and social exclusion, $1^{\text {a }}$ Ed., University of New South Wales, Sidney, Australia (2001)

Carvajal, N., Arrubla, M., y Caicedo, I., Educación financiera en los estudiantes de pregrado de la Universidad del Quindío, Sinapsis, 8(2), 99-120 (2016)

Duque, E.A., González, J.D., y Ramírez, J.D., Conocimientos financieros en jóvenes universitarios: caracterización en la Institución Universitaria Esemer, Revista de Pedagogía, 37(101), 41-55 (2016)

Fan, L., y Chatterjee, S., Financial socialization, financial education, and student loan debt, https://doi.org/10.1007/s10834-018-9589-0, J. Fam. Econ. Iss., 40, 74-85 (2019)

Federación Latinoamericana de Bancos, V Informe de inclusión financiera 2019, $1^{\text {a }}$ Ed., 122-123, Felaban, Buenos Aires, Argentina (2019)

Fernándes, D., Lynch, J.G., y Netemeyer, R.G., Financial literacy, financial education, and downstream financial behaviors, https://doi.org/10.1287/mnsc.2013.1849, Management Science, 60(8), 1861-1883 (2014)

Frisancho, V., The impact of financial education for youth, https://doi.org/10.1016/j.econedurev.2019.101918, Economics of Education Review, 78, 101918 (2020)

Garay, G., Índice de alfabetismo financiero, la cultura y la educación financiera, Perspectivas, (37), 23-40 (2016)

Grohmann, A., Klühs, T., y Menkhoff, L., Does financial literacy improve financial inclusion? Cross country evidence, https://doi.org/10.1016/j.worlddev.2018.06.020, World Development, 111, 84-96 (2018)

Harrington, C., Smith, W., y Bauer, R., Influencing business student intent to use a personal budget, J. Account. Educ., $26,135-153(2016)$

Iterbeke, K., De Witte, K., Declercq, K., y Shoelfhout, W., The effect of ability matching and differentiated instruction in financial literacy education. Evidence from two randomised control trials,

https://doi.org/10.1016/j.econedurev.2019.101949, Economics of Education Review, 78, 1-25 (2020)

Kaiser, T., y Menkhoff, L., Financial education in schools: a meta-analysis of experimental studies, https://doi.org/10.1016/j.econedurev.2019.101930, Economics of Education Review, 78, 101930 (2020)

Kaiser, T., y Menkhoff, L., Does financial education impact financial literacy and financial behavior, and if so, when? https://doi.org/10.1093/wber/lhx018, The World Bank Economic Review, 31(3), 611-630 (2017)

Kiyosaki, R., Despierta el genio financiero de tus hijos, 19a Ed., 43-50, Penguin Random House, Barcelona, España (2015)

Lusardi, A.M., Financial literacy and the need for financial education: evidence and implications, https://doi.org/10.1186/s41937-019-0027-5, Swiss J Economics Statistics, 155(1), 1-8 (2019)

Ministerio de Educación Nacional, Orientaciones pedagógicas para la educación económica y financiera, 1aㅡ Ed., MEN, Bogotá, Colombia (2014)

Moreno, E., García, A., y Gutiérrez, L., Nivel de educación financiera en escenarios de educación superior. Un estudio empírico con estudiantes del área económico-administrativa, Revista Iberoamericana de Educación Superior, 8(22), 163-183 (2017)

Ramos, J., Garcia, A., y Moreno, E., Educación financiera: una aproximación teórica desde la percepción, conocimiento, habilidad, y uso y aplicación de los instrumentos financieros, https://doi.org/10.17060/ijodaep.2017.n2.v2.888, International Journal of Development and Educational Psychology, (2), 267-278 (2017)

Roa, M.J., Garrón I., y Barboza J., Financial Decisions and Financial Capabilities in the Andean Region, https://doi.org/10.1111/joca.12187, J Consum Aff., 296-323 (2019)

Saldarriaga, C.A., Conocimientos financieros y capacidades financieras en colaboradores de una organización del Noreste Colombiano, Valor Contable, 5(1), 58-73 (2018)

Shim, S., Xiao, J.J., Barber, B.L., y Lyons, A.C., Pathways to life success: a conceptual model of financial well-being for young adults, https://doi.org/10.1016/j.appdev.2009.02.003, Journal of Applied Developmental Psychology, 30(6), 708723 (2009)

Silva, C.A., Celis, O., y Rangel, A.N., Impacto social de la educación financiera Caso: Uniminuto regional Bucaramanga, Santander Colombia, Boletín Redipe, 7(1), 116-123 (2018)

Superintendencia Financiera de Colombia, Actualidad del Sistema Financiero en Colombia, 1a Ed., 35-46, SFC, Bogotá, Colombia (2019)

Urban, C., Schmeiser, M., Collins, M., y Brown, A., The effects of high school personal financial education policies on financial behavior, https://doi.org/10.1016/j.econedurev.2018.03.006, Economics of Education Review, 78, 1-12 (2020)

Villada, F., López, J.M., y Muñoz, N., El papel de la educación financiera en la formación de profesionales de la Ingeniería, Formación Universitaria, 10(2), 13-22 (2017)

Xiao, J., y O'Neill B., Consumer financial education and financial capability, https://doi.org/10.1111/ijcs.12285, Int. J. Consum. Stud., 712-721 (2016) 